\title{
DIE ZUKUNFT DES KRIEGSRECHTS IM BEWAFFNETEN KONFLIKT*
}

Bewaffnete Konflikte prägen heute unübersehbar die innergesellschaftlichen Auseinandersetzungen zahlreicher Staaten der Dritten Welt. Dies gilt nicht allein, wie man zunächst annehmen würde, im Raum des Ringens um politische Selbstständigkeit, sondern gerade dort, wo dieses Ziel erreicht zu sein scheint: der Bürgerkrieg in Nigeria, der Zerfall Pakistans, die Umwälzungen in Kambodscha, die kriegerischen Auseinandersetzungen zwischen Vietnam und China stehen hier stellvertretend für viele bewaffnete Konflikte, die nach Gewinnung der politischen und staatlichen Unabhängigkeit ausgetragen wurden.

Ob diesem Phänomen neue, normverändernde Verhaltensweisen zugrundeliegen oder ob es nur die alten Interessengegensätze in verändertem Gewand widerspiegelt, ist schwer auszumachen. Tatsache ist, daß die Staatengesamtheit - trotz des Gewaltverbotes in den internationalen Beziehungen - bisher wenig Neigung zeigt, unmißverständlich und endgültig ihre Optionen für bewaffnetes Einschreiten in die innerstaatlichen Auseinandersetzungen anderer Staaten aufzugeben. Die Intervention zugunsten dessen, der sozialen Wandel mit Nachdruck verfolgt, wird offensichtlich immer noch als Teil oder als notwendige Begleiterscheinung von sozialem Wandel begriffen, den zu beeinflussen eine Säule klassischer Außenpolitik war und den man nicht ohne Zwang dem Reglement des Gewaltverbotes unterordnen möchte. Viele Staaten drängen zwar auf die erweiterte Anwendung des Gewaltverbotes auf bewaffnet ausgetragene innerstaatliche Auseinandersetzungen, jedenfalls soweit der internationale Frieden davon in Mitleidenschaft gezogen werden kann. Auf der anderen Seite scheuen sie sich aber, interne Auseinandersetzungen, trotz international nachteiliger Folgen, schon zum Anlaß zu nehmen, den Anwendungsbereich internationaler Verbotsnormen auszuweiten, die ja von der Sache mehr die Garantie des Status quo als den sozialen Wandel im Auge haben. Was auch immer die Intentionen sind, die einzelne Staaten an der Intervention festhalten lassen, unbestreitbar ist, daß alle Staaten sich der Gefahr bewußt sind, die sich für die Allgemeingültigkeit des Gewaltverbotes in seiner engeren Bedeutung, als Verbot der militärischen Gewaltanwendung und -androhung, ergeben, wenn Auseinandersetzungen im Innern auf die zwischenstaatlichen Beziehungen übergreifen.

Die Frage der völkerrechtlichen Relevanz innerstaatlicher bewaffneter Konflikte wird weiter dadurch kompliziert, daß im Grenzbereich zwischen innerstaatlicher und internationaler Rechtsordnung angesiedelte Konflikte von den in ihrer Herrschaftsposition Bedrohten seit jeher den ,,inneren Angelegenheiten“ der Staaten (domaine reservé) zugerechnet werden (wo internationale Ordnungsprinzipien traditionell keine Geltung besitzen), wenn es darum geht, politische Entscheidungsfreiräume zu erhalten oder sie stärker, als es übergeordnete internationale Rechtssätze gestatten, im Eigeninteresse auszunutzen. Nur im spezifisch humanitären Bereich erkennen die Staaten die Notwendigkeit gewisser allgemeinverbindlicher Regelungen an und unterwerfen sich entsprechenden internationalen Konventionen, wenn auch zögernd und mit mancherlei Vorbehalten, die ihre Effizienz häufig einschränken.

\footnotetext{
* Zugleich Besprechung von:

Aldo Virgilio Lombardi, Bürgerkrieg und Völkerrecht.

Die Anwendbarkeit völkerrechtlicher Normen in nicht-zwischenstaatlichen bewaffneten Konflikten. Schriften zum Völkerrecht Bd. 53, Duncker \& Humblot, Berlin 1976, 416 S.

Rolf Ottmüller, Die Anwendung von Seekriegsrecht in militärischen Konflikten seit 1945.

Das geltende Seekriegsrecht in Einzeldarstellungen, Bd. 10, hsg. von Prof. Dr. Herbert Krüger, Prof. Dr. Ingo v. Münch, Prof. Dr. Rolf Stödter, Alfred Metzner Verlag GmbH, Frankfurt/M. 1978, 375 S.

Law and Responsibility in Warfare, The Vietnam Experience. Ed. by Peter D. Trooboff, forworded by J. Goldberg. The University of North Carolina Press Chapel Hill 1975, 280 S.
} 
Die Frage also, in welchem Ausmaß innerstaatliche Veränderungsprozesse im Interesse der Staatengesamtheit einem internationalen Reglement unterstellt werden, ist nicht neu. Sie verdient jedoch auf dem Hintergrund eines zunehmend schärfer werdenden internationalen Verteilungskampfes neu gestellt und bedacht zu werden. Dazu geben die Veröffentlichungen von Lombardi, Ottmüller und der von Trooboff herausgegebene Band Veranlassung, die Fragen behandeln, die immer dann in den Vordergrund des Interesses rücken, wenn wirtschaftliche und soziale Defizite sich in bewaffnete Auseinandersetzungen entladen haben.

\section{Abstrakte Kriterien für die Bestimmung internationaler Rechte und Pflichten im inner- staatlichen bewaffneten Konflikt?}

Lombardi behandelt sein Thema, die Anwendbarkeit völkerrechtlicher Normen in nicht zwischenstaatlichen bewaffneten Konflikten, vorwiegend unter theoretischen Aspekten. Ausgangspunkt seiner Untersuchung ist die Erkenntnis (weniger die Erfahrung), daß in innerstaatlichen Konflikten, auch wenn sie Bürgerkriegscharakter angenommen haben, eine formelle Anerkennung der Aufständischen seitens der Mutterregierung oder eines dritten Staates heute nicht mehr ausgesprochen wird, womit eine wesentliche Voraussetzung der Anwendung des traditionellen Kriegsrechts entfallen ist. Da auch neueste vertragliche Regelungen des ius in bello keine verbindliche Definition des internen Konflikts geben ${ }^{\mathbf{1}}$, sieht Lombardi im Merkmal der ,Intensitätsstufen, die innerstaatliche Konflikte durchlaufen können"-er teilt sie ein in: Aufruhr-Aufstand-Bürgerkrieg-internationaler Krieg - sowie im Merkmal der „,Innerstaatlichkeit bewaffneter Auseinandersetzungen“ zwei Kriterien, mit deren Hilfe er das Abgrenzungsproblem ,,Krieg und Frieden“ "zu meistern glaubt. Dabei soll das Merkmal der Intensität der Auseinandersetzung den bewaffneten Konflikt nach unten, zum erlaubten und darum völkerrechtlich irrelevanten Konflikt, hingegen die Qualifikation der Innerstaatlichkeit den bewaffneten Konflikt nach oben, zur unbestritten verbotenen internationalen Gewaltanwendung abgrenzen helfen.

So sehr Lombardis Abgrenzungskriterien realistisch erscheinen, weil sie die Möglichkeit zu einer stärkeren internationalen Kontrolle der Methoden der Austragung innergesellschaftlicher Veränderungen eröffnen ohne den Staaten die Kompetenz für sozialen Wandel zu nehmen, so wenig konsequent führt Lombardi sein Vorhaben durch. Denn nur für die Bestimmung der Intensitätsstufen innerstaatlicher Konflikte läßt er objektive Maßstäbe gelten, die er aus der Wirklichkeit moderner militärischer Auseinandersetzungen ableitet (ohne aber diesem Anspruch gerecht zu werden). Für die Bestimmung der Innerstaatlichkeit von Auseinandersetzungen hingegen greift er auf das Kriterium der ,,Souveränität" zurück, was er damit begründet, daß ,,sich der Waffengang innerhalb eines souveränen Staates abspielen muß“ (S. 109). Mit der Souveränität führt Lombardi jenes Unterscheidungsmerkmal ein, von dem sich auch das Rechtsinstitut der völkerrechtlichen Anerkennung herleitet, das er selbst zuvor als für die Problemlösung ungeeignet eliminiert hat. Nun ist an sich gegen die Verwendung des Souveränitätsbegriffes nichts einzuwenden, wenn dieser über seine juristische Komponente hinaus auch soziologisch erfaßt würde. Aber gerade davon will Lombardi nichts wissen. Er erklärt punktum: ,,staats- und völkerrechtliche Abhängigkeiten können nur (sic!) in juristischen Kategorien ausgedrückt werden" (S. 110).

1 Art. 1 des 2. Zusatzprotokolls von 1977 zu Art. 3 der vier Genfer Konventionen von 1949 beschreibt nur formal einen Aspekt des internen Konflikts, indem er ,, dissident armed forces or other organized armed groups" heraushebt und in den weiteren Voraussetzungen näher bestimmt. Die ausdrücklich ausgenommenen ,, international disturbances", ,,tensions“, ,,riots“" etc. werden jedoch durch kein Kriterium voneinander und alle zusammen nicht vom ,,non-international armed conflict“" unterschieden. 
Den einmal eingeschlagenen Weg verfolgt Lombardi in seiner weiteren Erörterung mit erstaunlicher Realitätsferne. Das ist schon deshalb unverständlich, weil die gesamte Dekolonisierungspraxis der Vereinten Nationen, die auch er dem Leser nicht vorenthält, gerade den sozialen Vorgang in den Vordergrund der Problemlösung stellt und nicht die juristischen Aspekte, wie sie ihr - in der Gewähr der politisch-rechtlichen Selbstständigkeit - zugestandenermaßen auch zugrunde liegen. Die nichtjuristischen Aspekte des Dekolonisierungsvorgangs - sind so zwingend, daß Lombardi ihnen in der Darstellung des Abstimmungsverhaltens in den UN-Organen, der Berichte des UN-Generalsekretärs und der Stellungnahmen im Friendly relations-Ausschuß selbst nicht ausweicht (S. 242-272, 284-286, 290-298). Trotzdem verdienen seine ,Conclusiones“ diese Bezeichnung nicht, wenn dort apodiktisch erklärt wird: ,,Im innerstaatlichen Bereich können bewaffnete Auseinandersetzungen nicht verboten werden, da dies einer Garantie des Status quo gleichkäme. Die Gestaltung der innerstaatlichen Verfassungswirklichkeit muß ausschließlich dem Staatsvolk überlassen bleiben, wobei ihm auch ein revolutionärer Umsturz möglich sein muß.“ Ähnlich unergiebig sind Lombardis Folgerungen, wenn er feststellt, daß de facto-Regime dem Gewaltverbot unterliegen, sobald die konstitutiven Elemente der souveränen Staatlichkeit vorliegen - wahrlich keine überraschende Erkenntnis angesichts des Umstands, daß auch die UN-Charta nicht auf die De-jure-Anerkennung des Staates abstellt (Art. 2).

Es muß aber die Frage gestellt werden, ob die moderne Staatenpraxis nicht tatsächlich bestimmte Formen der innerstaatlichen Auseinandersetzung und der äußeren Einflußnahme auf sie aus dem gemeinsamen und übergeordneten Interesse aller Staaten an einer friedlichen Entwicklung außer Kraft gesetzt und welche anderen Verhaltensweisen sie an deren Stelle legitimiert hat. Die Antwort hierauf kann nicht allein in rechtlichen Kategorien gesucht werden. Dazu hätte es zumindest bedurft, die veränderten Verhaltensweisen auf der Ebene der UNO, gerade im Hinblick auf den traditionellen Souveränitätsbegriff, in die Untersuchung miteinzubeziehen. Solche veränderten Verhaltensweisen sind zu vermuten angesichts der Weiterentwicklung des UN-Friedenssicherunggedankens und zwar sowohl im unmittelbar die Vereinten Nationen interessierenden Bereich der Sicherung des De-facto-Friedens (z. B. durch peace keeping-operations) als auch im Bereich des indirekten Friedenssicherungssystems. Dazu gehören die zunehmend differenzierter werdenden Methoden des durch die UNO gesteuerten peaceful change, die zahlreichen Aktivitäten der UN-Organe und UNSonderorganisationen in wirtschaftlichen und sozialen Fragen ihrer Mitglieder als Ausdruck verstärkter Anstrengungen im Bereich der präventiven Friedenssicherung sowie das starke Gewicht, das die Vereinten Nationen und durch sie die Weltmeinung den Menschenrechten als einer neuen Kategorie in der internationalen Politik verschafft haben. Alle diese Aktivitäten und Praktiken beweisen, daß die Vereinten Nationen den Vorgang innergesellschaftlicher Veränderungen nicht mehr tabuisieren, sondern ihn in das internationale Sicherheitssystem zu integrieren suchen.

\section{Moderne Staatenpraxis als Indikator für veränderte Verhaltensweisen im bewaffneten Konflikt}

Mit der Arbeit Lombardis kontrastiert Ottmüllers vergleichende Untersuchung bewaffneter Konflikte der Nachkriegszeit (seit 1945). Obgleich Ottmüller sein Thema anders akzentuiert, indem er es auf die bewaffneten Auseinandersetzungen zur See konzentriert, ist auch sein Anliegen die grundsätzliche Frage, wie das Völkerrecht im bewaffneten Konflikt unserer Zeit, gekennzeichnet durch die Gemengelage innergesellschaftlicher Wandlungen, zwi- 
schenstaatlicher Rivalitäten, wirtschaftlicher und sozialer Defizite, alter und neuer Allianzen und globaler Macht- und Sicherheitsansprüche, gehandhabt, fortgebildet oder ersetzt worden ist.

Ottmüller geht von der Feststellung aus, daß die veränderten Grundnormen der Staatengemeinschaft, wie sie seit 1945 in der UN-Charter fixiert sind (Gewaltverbot, friedliche Sireitbeilegung, System der kollektiven Sicherheit, Selbstverteidigung mit Waffengewalt nur im Ausnahmefall), eine klare Einordnung überlieferter Rechtszustände (Krieg und Frieden) weithin nicht erlauben, und sucht deshalb seine Antworten nicht auf der theoretischen Ebene. Er erklärt, daß ,,einem Rechtssystem, welches in weit stärkerem Maße als andere Rechtssysteme von historischen, soziologischen, politischen wie technischen Faktoren abhängt, dessen Wertordnung zudem eher von einer Wertentleerung gekennzeichnet ist, ein solcher Weg wenig angemessen ist" (S. 2 f.). Ottmüller macht die Staatenpraxis und damit das objektiv darstellbare Geschehen zum Angelpunkt seiner Untersuchung. Seine praxisbezogene Vorgehensweise rechtfertigt er zusätzlich mit dem Argument, daß ein Umbruch in den tradierten Krieg-Frieden-Vorstellungen am nachhaltigsten in nicht typisch kriegsrechtlichen, aber doch militärischen Konfliktsituationen nachweisbar sein müßte, da diese ,,in weit stärkerem Maße als die Weltkriegspraxis Ausläufer eines klar und bewußt gesteuerten militärischen Vorgehens" seien (S. 3).

Obgleich Ottmüller eine spezifische Variante des bewaffneten Konflikts, die militärische Auseinandersetzung zur See, zum Thema hat, bezieht er immer den politischen Hintergrund der Konflikte in seine Untersuchung ein und schon gar nicht flüchtet er sich in die Abstraktion von Begriffen. Dagegen ist er schon durch seine Affinität zur amerikanischen realistischen Schule (McDougal) gefeit, von der er sagt, daß ihre Gedankengänge dem heutigen Konfliktgeschehen noch am ehesten gerecht werden könnten. Ottmüller sieht im bewaffneten Konflikt seit 1945 ein Konfliktfeld, gleichsam den Rahmen, in dem politische Organisationen, die ihren Befehlen Wirksamkeit verleihen und sich kriegsrechtmäßig verhalten können, anderen derartigen Organisationen gegenüber militärische Maßnahmen anwenden, ohne solche von der vorherigen Beobachtung überkommener Formvorschriften (Kriegserklärung, Anerkennungsakte) abhängig zu machen. Ottmüller gelingt es dadurch, Konfliktsituationen, in die innerstaatliche wie internationale Auseinandersetzungen ununterscheidbar miteinander verwoben sind, unter einer gemeinsamen Fragestellung zusammenzufassen und als Anschauungs- und Beweismaterial für normative Veränderungen zu präsentieren. Im Hauptteil seiner Arbeit beschreibt Ottmüller in chronologischer Reihenfolge die zwölf bedeutenden bewaffneten Auseinandersetzungen der Nachkriegszeit, im einzelnen: die Konflikte in Palästina 1948 und 1965 (1. und 2. Nahostkonflikt), in Suez 1956, in Algerien 1954/61, in Cuba 1962, zwischen Indien und Pakistan wegen der Zugehörigkeit Kaschmirs 1965, den Rhodesienkonflikt 1965/77, den Vietnamkonflikt 1964/75, den Sezessionskrieg in Nigeria (Biafrakonflikt) 1967/70, den indisch-pakistanischen Konflikt um den Abfall Bangla Desh's 1971, und den Oktoberkrieg (Yom Kippur-Krieg oder 4. Nahostkonflikt) 1973. Jeder dieser Konfliktfälle wird nach ein und demselben Schema abgehandelt: Historischer Uberblick, Konfliktverlauf und rechtliche Auswertung, unterschieden nach Praxis, Rechtsprechung, Schrifttum und eigener Stellungnahme. Ottmüller legt Gewicht auf die Frage, inwieweit die angewendeten Maßnahmen typisch kriegsrechtliche oder eher friedensrechtliche Elemente erkennen lassen. Für die Zuordnung der Maßnahmen zur ersten Kategorie legt er die Maßstäbe des überlieferten Kriegs- und Neutralitätsrechts an: Wahrung des Publizitätsprinzips, Einhaltung der zeitlichen und territorialen Schranken, Respektierung neutraler Flaggen, Wahrung blockaderechtlicher und konterbanderechtlicher Schranken, Kontrollformen, Einhaltung der Rechtsweggarantien (Ủberprüfung der Maßnahmen durch Prisen- 
gerichte), Berücksichtigung humanitärer Bestimmungen. Die Zuordnung zur zweiten Kategorie (friedensrechtliche Elemente der Maßnahmen) bestimmt er danach, inwieweit anstelle typisch kriegsrechtlicher Normen im Prinzip friedensrechtliche, darunter auch polizeirechtliche Kriterien die Praxis ausgerichtet haben.

Ottmüllers streng systematisierte Vorgehensweise beantwortet die Frage der tatsächlichen Einhaltung des Gewaltverbotes differenziert insoweit, als sie auch darüber Aufschluß gibt, welche Restriktionen sich die Staaten auferlegten, wenn sie das Gewaltverbot nicht konsequent beachtet haben. Dazu gehört die Feststellung, daß die Staaten den konfliktausdehnenden Formen der Auseinandersetzung, wie sie exemplarisch in der Quarantäneanordnung Präsident Kennedys während der Cuba-Krise zur Durchführung kamen, keine Absage erteilten, sofern nur die risikoerhöhenden Faktoren solcher Maßnahmen durch ein entsprechend differenziertes crisis management unter Kontrolle gehalten werden konnten.

In der Auswertung der vorbildlich recherchierten Konfliktfälle kommt Ottmüller zu dem Ergebnis, daß die moderne Staatenpraxis der rationalen Anwendung des überlieferten Kriegsrechts die optimistische These widerlege, derzufolge dem Kriegsrecht wegen des Gewaltverbotes keine rechtserhebliche Bedeutung mehr zukomme, wie sie auch umgekehrt die pessimistische These nicht bestätige, daß die heutige Waffentechnik, insbesondere die Massenvernichtungswaffen völkerrechtliche Schranken illusorisch erscheinen lasse. Der wesentliche Unterschied heutiger militärischer Konflikte zum Kriege, so erklärt der Autor, sei der, daß die Staaten letzte Mittel nur androhen, möglicherweise auch erwägen, aber nicht tatsächlich zur unberechenbaren Wirklichkeit machen. Auf diese Weise bleibe die Repressalienspirale außer Anwendung und es werde auf das überlieferte (See-)Kriegsrecht zurückgegriffen, hinsichtlich dessen Schranken und Pflichten nur geringe Meinungsverschiedenheiten bestünden (S. 308).

Richtig ist Ottmüllers Feststellung, daß die aus der UN-Charta resultierenden besonderen Befugnisse keine Entpflichtung vom traditionellen Kriegsrecht mit sich bringen. Auch ein erklärter Nichtanerkennungsvorbehalt sei für die Frage der Rechtsbindung unerheblich (S. 309 ff.). Allerdings haben die Vereinten Nationen bisher in keinem Fall traditionelles Kriegsrecht pauschal zur Anwendung gebracht, sondern ihre Maßnahmen in Anlehnung an die Leitprinzipien der UN-Charta auf die jeweilige Konfliktsituation abgestimmt, vor allem aber unter Berücksichtigung der politischen Entwicklungstendenzen getroffen. Die Vermutung liegt deshalb nahe, daß immer dann, wenn heute bewaffnete Auseinandersetzungen ausgetragen werden, die Beobachtung allgemeinverbindlicher Standards zum einen ein Gebot der Humanität ist, zum anderen aber auch Teil einer Auseinandersetzung ist, in der nicht nur um politische Ziele gerungen, sondern ebenso die Methoden und Bedingungen für künftige Auseinandersetzungen festgelegt werden. Dazu ist Voraussetzung nicht eine annähernde Waffengleichheit, aber eine gewisse Ubereinstimmung in der Auswahl der von beiden Seiten als geeignet angesehenen Kampfmethoden. Andernfalls ist das Gegenseitigkeitsprinzip, das jeder für verbindlich gehaltenen zwischenstaatlichen Norm zugrundeliegt, nicht gewahrt. In Vietnam geriet das Kriegsrecht aus diesem Grunde zeitweilig zwischen die Mühlsteine, nicht weil die militärischen Ausgangspositionen und das gegenseitige Kräfteverhältnis stark divergierten. Ottmüller darf sich deshalb bestätigt sehen, wenn er eine Tendenz zu einer weitgehenden Anwendung des tradierten Kriegsrechts im Bürgerkrieg, vor allem des Humanitätsrechts, unter drei Voraussetzungen feststellt: Das Konfliktgeschehen muß sich über eine längere Zeitspanne erstrecken, der Gegner muß (damit) über eine Organisation verfügen, die seinen Anordnungen Nachdruck verleiht (Prinzip der Effektivität), und die Organisation darf nicht von vornherein auf die Ausübung von Terror ausgerichtet sein. Davon abgesehen hält er in jedem Fall im bewaffneten Konflikt zwei Grundnormen für 
verpflichtend: die Aufrechterhaltung der Unterscheidung zwischen Kombattanten- und Zivilistenstatus sowie die Unterscheidung zwischen militärischen und nichtmilitärischen Objekten. Ob sich allerdings die verbleibenden Lücken des Kriegsrechts durch einen bloßen Appell, die bestehenden Normen zugunsten der Zivilbevölkerung erweiternd auszulegen, ausfüllen lassen, erscheint angesichts des Gewichtes, das Ottmüller selbst den Faktenfür das Zustandekommen von Normen beimißt, fraglich.

\section{Seerechtspraxis als Ausdruck einer friedensrechtlichen Orientierung im bewaffneten Konflikt}

In der interessanten Frage der völkerrechtlichen Qualität der Vermeidung kriegsrechtlicher und der Anwendung prinzipiell friedensrechtlicher Kriterien sind Ottmüllers Aussagen konkret genug, um die Seerechtspraxis in bewaffneten Konflikten als eine Neuformulierung traditionellen Kriegsrechts erscheinen zu lassen. Die Veränderungen sind dadurch gekennzeichnet, daß die Konfliktparteien sich in der Regel keine Ziele setzten, wie sie für die frühere Annexionspraxis typisch waren. Die moderne Staatenpraxis legt stärkeres Gewicht auf das Moment der Sicherheit und weniger auf Gerechtigkeit, was der Praxis des Sicherheitsrates exakt entspricht. Mit Recht deutet Ottmüller deshalb die Tatsache, daß die militärischen Aktionen nicht mehr mit dem Ziel der endgültigen Niederwerfung des Gegners verfolgt werden, insbesondere kein politisches Auslöschen beabsichtigen, als einen nach 1945 eingetretenen Wandel. Allerdings ist auch in dieser Hinsicht Vietnam nicht repräsentativ gewesen: Weder Hanoi noch Saigon oder Washington haben von Anfang an ihre politischen Ziele in Südvietnam anders als total verstanden, bis die Vereinigten Staaten im Angesicht der bevorstehenden Niederlage ihren Verteidigungsperimeter vom Festland wieder auf die vorgelagerte Inselkette zurücknahmen. Ob die USA im bewaffneten Konflikt mit Nordvietnam dem Kriterium der Vermeidung typisch kriegsrechtlicher Kriterien durch Einhaltung des Proportionalitätsprinzips Rechnung getragen haben, wie Ottmüller meint, gilt deshalb in dieser Unbedingtheit nicht uneingeschränkt. Zumindest zeitweise hat ihre Luftkriegspraxis gegen Nordvietnam (im ,,Christmas bombing“ 1972) deutlich Züge einer totalen Kriegführung gezeigt (s. unten S. 399 ff.). Allein die Tatsache, daß im Luftkrieg neben dem crisis management auch Gesichtspunkte der Verhältnismäßigkeit eine Rolle gespielt haben, weil sie einen Ausweg aus der politisch unerwünschten Eskalationsspirale aufzeigten, erlaubt noch keine Bewertung des Luftkrieges als einer am Friedenszustand orientierten Maßnahme.

Die von Ottmüllers beobachtete zeitliche Begrenzung der militärischen Konflikte charakterisiert die Staatenpraxis jedoch zu Recht als einen Grundzustand und damit als eher am Friedens- als am Kriegsrecht orientiert. Sie schloß regelmäßig die Inanspruchnahme prisenrechtlicher Befugnisse nach Abschluß eines Waffenstillstandes aus, trotz Art. 36 der Haager Landkriegsordnung. Die.USA und die UdSSR haben zwar keinen Widerspruch darin gesehen, im Nahostkonflikt die Parteien zur Feuereinstellung aufzufordern und sie gleichzeitig weiter mit Waffen zu beliefern. Doch war dieses Verhalten weniger Ausdruck ihrer Inkonsequenz als ihres gemeinsamen Interesses, über die Wiederherstellung des Status quo das bestehende Kräftegleichgewicht nicht einer neuen pluralistischen Ordnung zu opfern. Unter den sicherheitspolitischen Gegebenheiten und Ansprüchen der führenden Seemächte können jedenfalls militärische oder andere Kontrollmaßnahmen, die in der Vergangenheit noch gegenüber allen am Konflikt nicht beteiligten Schiffen ohne Unterschied zur Anwendung 
gelangt waren, nicht mehr uneingeschränkt als gültige Ausübung des Kriegs- und Neutralitätsrechts angesehen werden (S. 318-324).

Zum gleichen Ergebnis kommt Ottmüller in der Frage der Rechtsfolgen ergriffener Kontrollmaßnahmen: Anstelle der früher nach Prisenrecht verfügten entschädigungslosen Einziehung von Handelsschiffen tritt die Sequestration, die Schiffe und Güter nach den Grundsätzen des Seehandelsrechtes vorübergehend festhält und sie dem Beuterecht entzieht. Dies gilt jedenfalls für jene Konflikte, die, weil unterhalb der Schwelle einer mit Atomwaffen geführten Auseinandersetzung gehalten, eines ausgedehnten Kriegsschauplatzes entbehren (S. 324 ff.). Mit dieser Praxis werde, so erklärt Ottmüller, an die zahlreichen Friedensblockaden des 19. Jahrhunderts angeknüpft, die gleichfalls für die betroffene Handelsschiffahrt ohne prisenrechtliche Folgen blieben. Sie findet ihre Ergänzung in bestimmten innerstaatlichen Vorkehrungen, wie Lieferverbote, Genehmigungszwang für Exporte etc., was ihre friedensrechtliche Orientierung deutlich herausstellt.

Für die Behandlung der nichtmilitärischen Güter, die nach traditionellem Kriegsrecht einer strengen konterbanderechtlichen Úberprüfung unterlagen, später aber immer mehr einer pauschalen entschädigungslosen Einziehung unterworfen wurden, gilt heute prinzipiell nichts anderes. Die Begrenztheit der politischen und militärischen Ziele läßt die konterbanderechtlichen Kriterien hinter andere Ưberlegungen zurücktreten: Nicht mehr der Beuteaspekt, sondern ausschließlich die Relevanz von Waren für die konkrete Konfliktführung bestimmen Umfang und Zusammensetzung der indizierten Güter. Auch der Gesichtspunkt, wieweit im Falle einer Freigabe der Grundsatz der Gegenseitigkeit gewahrt erscheint, rückt die Seerechtspraxis in bewaffneten Konflikten näher an das Friedensrecht als an das Kriegsrecht. Auf der anderen Seite sind formell beschlagnahmte Waren in der Regel keiner prisenrechtlichen Uberprüfung unterzogen worden. Jedenfalls ist nur in zwei von zwölf Fällen (im Palästinakonflikt und im indisch-pakistanischen Konflikt 1965), dabei in einem Fall nur von einer der beiden Parteien (Ägypten), ein Prisengericht eingesetzt worden. Allerdings geht Ottmüller der interessanten Frage, ob dieses Ergebnis mit der Tatsache in Verbindung gebracht werden kann, daß überwiegend bürgerkriegsähnliche Auseinandersetzungen die Nachkriegskonflikte bestimmt haben, nicht nach.

\section{Legitimiert das Völkerrecht die neuen Formen und Methoden der Gegenwehr im be- waffneten innerstaatlichen Konflikt?}

Anders als bei Lombardi und Ottmüller will der von Trooboff editierte Sammelband ,,Law and Responsibility in Warfare" nicht primär Fragen der Weitergeltung des Völkerrechts im bewaffneten Konflikt zur Diskussion stellen, sondern die durch die Erfahrungen im Vietnamkonflikt sehr viel pointierter gestellte Frage erörtern, in welchem Maße im Antiguerillakrieg eingesetzte Kombattanten die Kampfhandlungen noch in Ubereinstimmung mit dem Völkerrecht zu halten vermochten. Diese Frage nach den subjektiven Möglichkeiten, bestimmte Minimalstandards des ius in bello nicht zu unterschreiten, wird an den beiden spektakulärsten und umstrittensten Praktiken der Antiguerillakriegführung in der Gegenwart, dem von den USA in Südvietnam angewandten ,, Counterinsurgency programme" und dem sog. ,, Christmas bombing“" gegen Nordvietnam, exemplifiziert und durch Autoren der unterschiedlichsten Position und Herkunft in Rede und Gegenrede diskutiert. Die Fragestellung knüpft an Probleme der Kriegführung an, die bereits der amerikanische Bürgerkrieg, die Anfang des Jahrhunderts auf den Philippinen angewandte Kriegführung und gewisse barbarische Erscheinungen des Zweiten Weltkrieges (Flächenbombardements, Einsatz von 
Napalm, Massenexekutionen) aufgeworfen hatten. Gerade in der historischen Perspektive erscheint einiges von dem vorweggenommenen, was später in Vietnam in mehr oder weniger abgewandelter Form zur Anwendung gebracht wurde. Die Fragestellung knüpft damit auch an ein Kriegsrecht an, das die in Vietnam praktizierten Antiguerillakampfmethoden prinzipiell erfaßt hatte, lange bevor es überhaupt zu einem bewaffneten Konflikt in Vietnam kam. So bestimmte der „Lieber Code“, eine Kodifikation des Kriegführungsrechts, welche die USA 1863 offiziell als ,,Instructions for the Government of Armies of the United States in the Field" übernommen hatten, in Art. 15: ,Men who take up arms against one another in public war do not cease on this account to be moral beings, responsible to one another and to God".

Die Publikation dient der Auseinandersetzung um Inhalt und Grenzen der persönlichen Verantwortung und zwar innerhalb der Anfang der 70er Jahre verstärkt geführten öffentlichen Kontroverse um die Legitimität des amerikanischen Engagements in Südostasien. Dadurch gewinnt sie zentrale Bedeutung auch für die Frage der Weitergeltung des Kriegsführungsrechts unter den Bedingungen der modernen Guerilla und Antiguerilla. Drei Probleme markieren den Fragenkomplex: 1. Ist es völkerrechtlich zulässig, im Kampf gegen primitiv ausgestattete Aufständische das ganze Gewicht einer modernen Kriegstechnologie zur Anwendung zu bringen? 2. Verbietet das Gewohnheitsrecht die Anwendung von Tränengas, Napalm und Entlaubungsmitteln? und 3. Wieweit reicht die individuelle Verantwortlichkeit von Kombattanten und von Personen, die Befehlsgewalt haben?

\section{Völkerrechtlich problematisches „,Counterinsurgency“}

Zum zentralen Fragenkomplex des technologischen Ubergewichts der USA in der Guerillabekämpfung liefert Richard Falk die Hauptstichworte. Er sieht keine Rechtfertigung für das Argument, wie es von dem amerikanischen Theologen Paul Ramsey gebraucht wurde, daß in einem Krieg, der bereits durch den selektiven Terror der Aufständischen gegen zivile Amtsund Funktionsträger brutalisiert worden ist, die volle Verantwortung für indiskriminierende Gegenmaßnahmen die Rebellen trifft. Falk hält eine sich primär gegen die Zivilbevölkerung auswirkende Strategie, die die ganze Palette der modernen Kriegstechnologie zum Einsatz bringt, für eine Form des Genocids und ein Verbrechen gegen die Menschlichkeit schlechthin. Der unbeschränkten Kriegführung solchen Zuschnitts setzt er vier grundlegende und als ius cogens allgemeinverbindliche Prinzipien entgegen: Das Gebot, unnötige Leiden zu vermeiden (Grundsatz der Notwendigkeit der Maßnahmen), das Gebot, zwischen militärischen und nichtmilitärischen Zielen zu unterscheiden (Diskriminierungsgebot), das Gebot, die Verhältnismäßigkeit der Mittel zu den Zielen zu beachten (Proportionalitätsprinzip) und das Gebot, den Mindeststandard unter Humanitätsprinzipien zu wahren. R. Falk räumt zwar ein, daß das ,,fact-finding“" als Voraussetzung einer rechtlichen Bewertung von Counterinsurgency-Methoden problematisch sei, sieht jedoch diese Probleme durch jene, die die zugrundeliegende Politik selbst nicht in Zweifel ziehen, überbewertet. Er glaubt den Hauptmangel der Counterinsurgency-Strategie in Vietnam im massiven Einsatz der Luftwaffe zu erkennen, welche ein Höchstmaß an Schonung amerikanischen Menschenlebens bei einem Maximum an Zerstörungswirkung auf der gegnerischen Seite (oder was man dafür hielt) garantierte. Bindeglied zwischen beiden Zielen war die Unterstellung, die Zivilbevölkerung arbeite mit den Aufständischen zusammen, was die Kritiklosigkeit erklärt, mit der die Zerstörung der Infrastruktur im Rahmen des ,,Phoenix-Programms“ (Vernichtung der Ernten, Zerstörung oder Verunreinigung der Brunnen, Verwandlung landwirtschaftlicher 
Flächen in Odland u. a.) hingenommen wurde. Gleiches gilt für die Einrichtung sog. ,,Free fire-Zones" in Gebieten, die durch administrativen Akt zu Feindgebieten erklärt wurden. Sie erlaubte es den US-Einheiten nicht nur, auf alles zu schießen, was sich bewegte, sondern auch die Opfer unterschiedslos als , ausgeschaltete Feinde“ statistisch zu erfassen. Die Folge von all dem war ein Zahlenmißverhältnis von 10:1 zwischen zivilen und militärischen Opfern, das die ganze Fragwürdigkeit einer Strategie offenbarte, die nicht den Feind identifizierte, um ihn auszuschalten, sondern die Bewohner ganzer Regionen vorweg und pauschal zu Feinden erklärte und den militärischen Erfolg durch die Zahl der Opfer in diesen Regionen definierte.

Falk sieht eine Ursache für das Mißverhältnis zwischen militärischen und zivilen Opfern in der Außensteuerung des Counterinsurgency-Programms, d. h. in der Tatsache, daß eine von den nachteiligen Folgen direkt nicht betroffene Macht Entscheidungskompetenz erhielt, wodurch die Interessen der vietnamesischen Zivilbevölkerung tendenziell stärker den militärischen Zwecken untergeordnet wurden. Eine andere Ursache sieht Falk darin, daß die brutalen Methoden des Counterinsurgency als Substitut für die mangelnde Unterstützung der Bevölkerung im Kampf gegen die Rebellenbewegung verstanden wurden. Falk stellt deshalb nicht nur eine pessimistische Prognose, wenn er den Staaten noch viele Kriege des Typs Vietnam voraussagt. Die Dehumanisierung des individuellen Verhaltens liege, so erklärt er, in der Logik des Counterinsurgency. Sie könne nur aufgehalten werden, wenn der Forderung nach einer strikteren Einhaltung des ius in bello eine grundlegende Neubestimmung der weltweit praktizierten Antiguerilla-Kriegführung vorausgeht.

\section{Counterinsurgency ohne individuelle Haftungsfolgen?}

R. Falks Sicht der Dinge wird von den anderen Autoren nicht geteilt. R. E. Jordan III glaubt aufgrund der mangelnden Präzisierbarkeit elementarer Rechtsbegriffe, wie ,,militärisches Erfordernis“ (Präambel zur IV. Haager Konvention von 1907), oder aufgrund der Zweideutigkeit der Situationswirklichkeit den Kombattanten von individuellen Haftungsfolgen weitgehend freigestellt. Etwas undifferenziert und unkritisch setzt er die spezifischen im Vietnamkrieg praktizierten Counterinsurgency-Methoden mit der ,, in jedem Krieg beobachteten Verletzung der Kriegführungsregeln" gleich oder sucht sie als vietnamesische Variante des überall und zu allen Zeiten vorrangigen Úberlebenswunsches der Kombattanten zu erklären. Jordan räumt zwar ein, daß die „,Free fire Zones“ selbst nicht im Einklang mit den Vorschriften standen, wie sie von der militärischen Führung der USA 1965 erlassen worden waren. Er sieht jedoch die Mängel mehr in der technischen (und daher verbesserungsfähigen) Handhabung des Counterinsurgency (z. B. in den schwerfälligen und zeitraubenden ,Preclearences“ dünn besiedelter Gebiete) und weniger im System, das er selbst nicht in Frage stellt.

R. Baxter glaubt einen entscheidenden Mangel der amerikanischen Antiguerillakriegführung darin zu erkennen, daß die Haager Regeln auf internationale Konflikte Anwendung finden, während der Bürgerkrieg in Vietnam seine Beurteilung nur unter den Miniaturregeln des Art. 3 der Genfer Konventionen von 1949 finden könne. Angesichts des Umstands, daß die Counterinsurgency-Methoden von außen gesteuerte Bürgerkriegsstrategie war, ist Baxters Wertung indessen nur partiell wirklichkeitsgerecht. Auch sein Vorwurf, R. Falk vermenge rechtliche mit rechtspolitischen Erwägungen, trifft das Problem nicht, da die von Falk zentral herausgestellten vier Grundprinzipien in zahlreichen Vorschriften des Völker- 
gewohnheitsrechts und des Völkervertragsrechts als allgemeiner Grundgedanke des Völkerrechts nachgewiesen sind.

Wie Falk erkennt auch H. DeSaussure die prohibitiven und begrenzenden Wirkungen des traditionellen ius in bello für die moderne Antiguerillakriegführung an, sieht aber in der technologischen Úberlegenheit der einen Seite allein noch kein Manko, das automatisch den Rechtsverstoß begründet. Auch beurteilt DeSaussure die Rechtsstellung des von außen Intervenierenden nicht schwächer als die der autochthonen Bürgerkriegspartei, die von außen Hilfe in Anspruch nimmt. Schärfer als Falk allerdings differenziert DeSaussure innerhalb der vier Prinzipien, von denen er das der (militärischen) Erforderlichkeit und das der Menschlichkeit für die Basisprinzipien erklärt, die allen anderen Kriegführungsregeln zugrundeliegen. Beide korelieren miteinander, so daß ein ,,kalkuliertes “ Vorgehen, das unnötige Leiden auf der Seite des Feindes oder der Zivilbevölkerung verursache, immer auch militärisch überflüssig und unmenschlich sei. DeSaussures Folgerungen sind aber nicht konsequent, wenn es um ihre Umsetzung in die Praxis geht. So wertet er jeden Ort, der den Rebellen als Lager für Material, Truppen oder Nachschub dient, als angreifbares Ziel. Wenn aber der Schutz der Dörfer Aufgabe der Regierung ist, der Arm der Regierung andererseits nicht so weit reicht, um diesen Schutz zu gewährleisten, widerspricht es allen Grundsätzen des Rechts, die Dorfbevölkerung für die Unfähigkeit der Regierung haften zu lassen.

Vollends zynisch wird DeSaussures Argumentation dort, wo er erklärt, die „,Free Fire-Zones" seien das wirksamste Instrument der Guerillabekämpfung und im gleichen Atemzug die Zwangsläufigkeit der zivilen Opfer mit der mangelnden Identifizierbarkeit der Guerilla und ihrer Situation begründet. Wenn der Feind sich nicht offenbart, so etwa muß geschlossen werden, soll nicht die Effektivität der Antiguerilla-Strategie in Frage gestellt, sondern eine Haftungsumkehr gefordert sein. Die Haftung der Verantwortlichen wird damit durch eine solche der Zivilbevölkerung ersetzt (S. 73).

\section{Zur Illegitimität der Terrorangriffe}

Weniger kontrovers sind die Argumente in der Frage der rechtlichen Schranken des Luftkrieges DeSaussure und R. Glasser unterscheiden zunächst, ausgehend von der Praxis im Zweiten Weltkrieg und im Koreakrieg, fünf Arten des Luftkrieges: 1. Der Luftkrieg gegen militärische Ziele im engeren Sinne, wie Truppen, Ausrüstung, Depots, u. a.; 2. Der Luftkrieg gegen Transporteinrichtungen, Verkehrsverbindungen und wirtschaftliche Unternehmen, die sowohl militärischen als auch zivilen Zwecken dienen; 3. Der Luftkrieg gegen Ziele rein wirtschaftlichen Charakters, die nur indirekt einen Einfluß auf die militärische Stärke ausüben; 4. Terrorangriffe gegen zivile Ziele; 5. Repressalien. Die Autoren lassen keinen Zweifel daran, daß die zwei zuletzt genannten Formen des Luftkrieges heute völkerrechtlich unzulässig sind. Für die Terrorangriffe ergibt sich dies aus dem Grundsatz des Kriegsvölkerrechts, der die Kriegführung auf die Schwächung der militärischen Schlagkraft des Gegners beschränkt. Das Repressalienverbot ist unmittelbarer Ausdruck des Gewaltverbotes entsprechend dem heutigen Verständnis von der Gestaltung der staatlichen Zwischenbeziehungen. Im Falle der Flächenbombardements gegen deutsche und japanische Städte (Dresden, Tokyo z. B.) wird die Illegalität des Luftkrieges allerdings nicht ohne weiteres eingestanden mit Rücksicht auf eine ,,Nichtunterscheidbarkeit“ $z$ wischen militärischen und zivilen Zielen. Andererseits räumen DeSaussure und Glasser ein, daß auf britischer Seite gegenüber Deutschland und im Falle der Atombombenangriffe auf Hiroshima und Nagasaki die feste Absicht bestanden hatte, die Moral der Zivilbevölkerung zu lähmen. 
Das Problem, das sich im Zusammenhang mit den Bombenangriffen gegen Nordvietnam Weihnachten 1972 stellt (sog. „Christmas bombing“), ist insofern komplizierter, als die USA die Angriffe formal mit der Gewinnung militärischer Vorteile begründet hatten, während eine starke Vermutung dafür bestand, daß sie ausschließlich darauf abzielten, Nordvietnam zu einer politischen Verhandlungslösung zu zwingen. DeSaussure und Glasser sehen zwar das Dilemma, sobald die Doktrin der militärischen Notwendigkeit getrennt von den politischen Absichten, denen erstere regelmäßig untergeordnet ist, gesehen wird. Dennoch halten sie daran fest, daß Bombardements militärisch intendiert sein müssen und daß eine militärische Begründung ausreichend ist, solange die Angriffe deutlich, entscheidend und voraussehbar die militärische Schlagkraft zu treffen vermögen. Unmißverständlich sind hingegen die anderen Aussagen der Autoren zum Luftkrieg: Selbst wenn die rechtlichen Voraussetzungen für Luftangriffe gegeben sind, sind sie doch unzulässig, wenn die Ziele in dichtbevölkerten Regionen liegen und Luftangriffe incidenter unverhältnismäßige Schäden zu Lasten der Zivilbevölkerung (Hospitäler, Deiche etc.) verursachen. In jedem Fall, so erklären DeSaussure und Glasser, verbieten sich Luftangriffe zum Zwecke der Beschleunigung eines Verhandlungsfriedens selbst dann, wenn ausschließlich militärische Ziele angeflogen werden. $\mathrm{Zu}$ Recht heben sie hervor, daß ein unter dem Völkerrecht legalisiertes „,bombing for political ends“ den Luftkrieg von allen Schranken lösen und seine Ziele und sein Ausmaß dem subjektiven Ermessen anheimstellen würde.

Halten sich diese Feststellungen weitgehend im Rahmen dessen, was sinngemäß in die 1977 angenommenen Zusatzprotokolle zu den Genfer Konventionen von 1949 eingegangen ist (in das II., die nicht-zwischenstaatlichen Konflikte behandelnde Protokoll etwas weniger als in das I. Protokoll), so finden sie doch keine ungeteilte Zustimmung bei den anderen Autoren des Sammelbandes. Während Townsend Hoopes den beiden vorgenannten Autoren in der Bewertung des Christmas bombing von 1972 noch zu folgen bereit ist, hält er die Aussagen über das grundsätzliche Verbot des Luftkrieges mit politischenZielsetzungen für zu weitgehend. Er meint, die Strategie der amerikanischen Luftangriffe im Zweiten Weltkrieg war im breitesten Maße politischer Natur und habe sich aus der Totalität des Krieges selbst gerechtfertigt. Daher könne die amerikanische Strategie als die humanere Alternative verteidigt werden verglichen mit einer, die der japanischen Kriegsmaschinerie nur mit einer Kombination begrenzter konventioneller Angriffsmaßnahmen entgegengetreten wäre.

Die Argumentation, die den Einsatz von Massenvernichtungswaffen gegen die Zivilbevölkerung aus der zeitlichen Verkürzung des Krieges rechtfertigt, ist wiederholt gebraucht worden, ohne die entscheidenden Bedenken gegen sie ausgeräumt zu haben. Zwar ist dem Kritiker DeSaussures und Glassers recht zu geben, wenn er an den Mangel eines allgemeinverbindlichen systematischen Luftkriegskodex erinnert oder darauf verweist, daß das politisch motivierte Christmas bombing von 1972 keine Einzelerscheinung gewesen sei und insoweit auch den Nachweis verbindlicher Rechtssätze erschwere. Hoopes kommt aber nicht an der Tatsache vorbei, daß bereits die Land- : nnd Seestreitkräfte im 1. Weltkrieg seit Einführung der Haager Kriegsführungsregeln im Jahre 1907 gehindert waren, die Zivilbevölkerung aus politischen Ưberlegungen, etwa um die Kampfhandlungen abzukürzen, zum Ziel von Angriffen zu machen. Den Luftkrieg gegenüber der klassischen See- und Landkriegführung zu privilegieren, hieße nicht nur, ihm eine Qualität beimessen, die ihm von seiner Funktion her, klassische Kriegführung zu ergänzen, nicht zukommt, sondern würde auch den Intentionen des Kriegsvölkerrechts, grundlegende Prinzipien zur Begrenzung des Krieges in jedem Fall zu erhalten, entgegenwirken. Ob das Sicherheitsinteresse der Nuklearmächte auf dem Hintergrund der von beiden Seiten verfolgten Abschreckungsstrategie nach einer freieren Handhabung der Zerstörungskapazitäten zu Lasten der Zivilbevölkerung verlangt, ist eine 
Hypothese, deren Wirklichkeitsgehalt bisher nicht getestet wurde. Nachgewiesen ist demgegenüber, daß mit der Totalisierung des Krieges die Sicherheit objektiv nicht verstärkt wird.

Deshalb erinnert Hoopes fatal an die Recht mit Politik vermengende Gedankenwelt McDougals, wenn er erklärt, Moralfragen würden im Zusammenhang mit den Methoden, die im Luftkrieg angewendet werden, nur als relativ bewertet in dem Maße, in dem die Gerechtigkeit des konkreten Krieges als stark empfunden werde. Nur dort, wo es an entsprechenden Überzeugungen fehle, würden überhaupt die jede Kriegshandlung begleitenden Grausamkeiten gesehen und beklagt (S. 142). Hoopes ist entgegenzuhalten, daß die ,,Gerechtigkeit der Sache"schon in klassischer Zeit, als der Krieg nur um einer iusta causa wegen geführt werden durfte, kein geeignetes Kriterium war, um das Problem der ausufernden Kriegführung zu lösen. Sie ist es heute weniger denn je, nachdem die Lehre vom gerechten Krieg in der ersten Hälfte unseres Jahrhunderts noch einmal eine Renaissance erlebt hat, die in ihrer barbarischen Konsequenz nur von wenigen Kriegen vor Einführung des ius in bello erreicht worden ist. Auf diesem Hintergrund ist die von Hoopes geäußerte Erwartung, das Kriegsrecht habe größere Chancen der Verwirklichung im konventionell begrenzten Krieg und im Guerilla-/Antiguerillakrieg, wenig realistisch. Die Schutzwirkung humanitärer Normen beruht wesentlich auf der Anerkennung der absoluten Integrität der nichtkämpfenden Personen und der zivilen Sachwerte. Eine geteilte Integrität nach dem Muster ,Schutz im konventionellen Krieg - kein Schutz im totalen Krieg“" würde zwangsläufig die der Norm zugrundeliegenden ideellen Ưberzeugungen, von denen humanitäre Normen stärker als andere Normen des Völkerrechts abhängig sind, zerstören.

\section{Die Waffen im Antiguerilla-Krieg}

Weniger ausführlich als die Frage der Methoden im Antiguerilla-Krieg behandelt der Sammelband die Frage der Waffen. Das Problem wird jedoch schon weitgehend dadurch eingegrenzt, daß die Kriegführung des Counterinsurgency nur drei Waffen ins völkerrechtliche Zwielicht gebracht hat: das Tränengas, den Flammenwerfer (Napalm) und die Herbiziden (Entlaubungsmittel). Diese Waffen sind von 1965 an in Südvietnam angewandt worden - allerdings nicht alle dort zum ersten $\mathrm{Mal}$ - und stellten im besonderen Maße das ius in bello in Frage im Hinblick auf seine Tragfähigkeit als Instrument einer rechtlich begrenzten Kriegführung.

In seinem einleitenden Beitrag stellt zunächst Howard S. Levie diese drei Waffensysteme in ihrer unterschiedlichen Wirkungsweise vor. Danach besteht die Besonderheit des Tränengases darin, daß es zunächst aus humanitären Gründen im Counterinsurgency Anwendung fand, in erster Linie, um Zivilpersonen, die unter scharfen Beschuß zu geraten drohten, aus Tunneln, Verstecken u. ä. zu vertreiben oder auch, um Verwundete aus Kampfzonen auszufliegen, daß es aber dann auch offensiv eingesetzt wurde zur Bekämpfung der Aufständischen, um sie direkt unschädlich zu machen, um ihnen ihre Operationsbasis zu nehmen oder auch zu Aufklärungszwecken. Dem Napalm, den im 2. Weltkrieg verwendeten Phosphorbomben verwandt, bescheinigt Levie im Buschkrieg sehr hohe Effektivität, weshalb es in Südvietnam mehr als jede andere Waffe panikartige Reaktionen auslöste. Die Herbiziden endlich, wiewohl schon seit 1945 bekannt, gehören zu den Waffen, die erstmals in Vietnam zum Einsatz kamen. Mit ihnen wurden heimliche Infiltrationswege der Rebellen aufgedeckt, unübersichtliches Gelände entlang der Verkehrswege und Flüsse gegen Angriffe aus dem Hinterhalt transparent gemacht und der Gegner zu Positionsveränderungen gezwungen. 
Ein weiterer Verwendungszweck des Einsatzes von Herbiziden war der, die Aufständischen von der Versorgung mit Grundnahrungsmitteln abzuschneiden. Mit dem Tränengas sieht Levie eine Gemeinsamkeit der Herbiziden darin, daß beide zu ,friedlichen“ Zwecken ebenso wie als Kampfmittel eingesetzt werden können, was ihre generelle Diskriminierung erschwere. Auch bestehe eine definitorische Schwierigkeit darin, daß die Nuklearwaffen bisher keinem generell gültigen Verbot unterliegen. Wäre es anders, meint Levie, könnte ein Verbot des Gebrauchs bestimmter Gase in bewaffneten Konflikten unschwer in ein allgemeines Kriegsverbot umgedeutet werden. So stellt sich für Levie die Frage der rechtlichen Zulässigkeit weniger als ein Problem dar, das unter den bestehenden Regelungen, des Genfer Gas-Protolls von 1925, dem die USA erst 1/2 Jahrhundert später beigetreten sind, und der Haager Regeln von 1907, zu sehen und zu lösen wäre, sondern eher als ein Problem der politischen Opportunität, ob und inwieweit die mit diesen Waffen verfolgten militärischen Ziele akzeptiert werden können.

Robert W. Tucker will das Problem wieder auf den Boden klassischer Rechtsinstitute zurückholen, wenn er auf den Sinn und Zweck des ius in bello abstellt. Er betont, daß es nicht Aufgabe des Kriegsrechts sei, die für die Verfolgung eines bewaffneten Konfliktes geeigneten Waffen zu begrenzen, sondern solche Waffen zu bannen, die heimtückisch und inhuman sind, weil sie unnötige Leiden verursachen oder indiskriminierend wirken, d. h. nicht zwischen militärischen und nichtmilitärischen Personen und Sachen unterscheiden. Tucker sieht die völkerrechtliche Fragwürdigkeit einer Waffe als gegeben an, wenn die genannten Kriterien nicht nur in Spezialbestimmungen des Völkergewohnheitsrechts und des Völkervertragsrechts, sondern ebenso in prinzipiellen gewohnheitsrechtlichen Verboten des Kriegsvölkerrechts nachgewiesen werden können. Die Schwierigkeit glaubt Tucker jedoch nicht in der Ermittlung der Rechtsgrundsätze, sondern in ihrer Anwendung zu erkennen, wenn es darum geht, den Rechtsstatus der Waffen definitiv zu bestimmen. Er sträubt sich dagegen, die Humanität als eines der Anwendungskriterien für die Legalität oder Illegalität von Waffen in ihrer historisch gewachsenen Bedeutung als allgemeingültiger sittlicher Standard für zwischenstaatliches und zwischenmenschliches Verhalten gelten zu lassen, sondern will sie unter Heranziehung des Grundsatzes der Verhältnismäßigkeit als eine von den militärischen Zwecken abhängige Größe bestimmen. Nur diejenige Waffe sei, so erklärt er, als illegal anzusehen, die, am militärischen Zweck gemessen, unproportionale Leiden oder Zerstörungen hervorrufe (S. 162).

So sehr Tucker zuzustimmen ist, wenn er die militärisch ineffektive Waffe eo ipso als inhuman wertet, so wenig kann ihm in der Umkehr dieses Satzes gefolgt werden. Denn Tucker gelangt durch die enge Bindung des Kriteriums der Humanität an die Kriegsziele fast zwangsläufig zu einer pauschà ${ }_{\text {len }}$ Exkulpation aller neuen Waffen, sofern nur der militärische Nutzen zuvor hoch genug veranschlagt worden ist. Es liegt auf der Hand, daß eine derartige Relativierung des ius in bello nicht nur im Widerspruch zum geltenden Recht steht, sondern dieses selbst zerstört. Auch die von Tucker an sich richtig gesehene scharfe Trennung der Frage in eine nach der Legalität der Waffen und eine nach der Legalität ihres Gebrauches veranlaßt ihn nicht dazu, dem ius in bello im politisch-militärischen Entscheidungsablauf den Rang zu geben, der ihm von der Staatenpraxis traditionell zugestanden worden ist. Er meint, wie auch Hoopes (S. 142), daß die Grausamkeit primär im unpopulären (ungerechten) Krieg empfunden wird, und übersieht dabei, daß gerade der unpopuläre Krieg die Minimalstandards geschaffen hat, weil er im besonderen Maße zu extremen Verhaltensformen verleitet. 
Die Schwächen der Levieschen und insbesondere der Tuckerschen Argumentation werden auch von Anthony d'Amato schonungslos aufgedeckt, Er erklärt, daß das Verbot der chem. Waffen dann nur für die Zukunft als Forderung erhoben und nicht auch für die Vergangenheit als geltendes Recht festgestellt werden kann, wenn man, wie es Levie und Tucker tun, einen klaren Trennungsstrich zwischen Politik und völkerrechtlichen Pflichten der Staaten zieht und diese Pflichten vorzugsweise im Vertragsrecht sucht. Zu einem ganz anderen Ergebnis komme man jedoch, sobald man anerkenne, daß die Politik regelmäßig in einem Bereich formuliert werde, für den das Völkergewohnheitsrecht bereits traditionell Maßstäbe gesetzt hat. D'Amato weist nach, daß jedenfalls dort, wo die politische Entscheidung unmißverständlich war, sie auch in Úbereinstimmung mit den Regeln des Völkergewohnheitsrechts stand, das gerade auch die Zusammenfassung vieler politischer Entscheidungen der Vergangenheit in Situationen war, die sich nicht grundlegend von der in Vietnam unterschieden. D'Amato räumt zwar ein, daß rechtspolitische Forderungen nicht mit dem geltenden Recht gleichgesetzt werden dürfen. Auf der anderen Seite meint er aber, daß sich bei der Bestimmung des Inhalts einer rechtlichen Norm, wie dies auch die richterliche Spruchpraxis beweise, ,,Is“ und ,,Ought“ überschneiden (S. 178). D’Amato sieht den Grund für die ungerechtgefertigte scharfe Trennung von ,,Policy“ und ,,Legal Obligations of States“ in der unbewußten Furcht, möglicherweise selbst in die Lage zu geraten, für politische Entscheidungen zur Verantwortung gezogen zu werden. Er hält es deshalb auch nicht für nützlich, den Bereich der Staatenhaftung mit dem der Individualhaftung zu verschmelzen.

\section{Die Individualhaftung im bewaffneten innerstaatlichen Konflikt}

Obgleich d'Amato mit seinen Fragen zum Verhältnis der Individualhaftung zur Staatenhaftung Probleme aufzeigt, über die das völkerrechtliche Schrifttum gemeinhin hinweggeht, behandelt auch der Sammelband in dem Abschnitt, der der Individualhaftung gewidmet ist, diese Probleme nicht. In dem einleitenden Beitrag Paul C. Warnkes wird die Notwendigkeit einer scharfen Trennung zwischen ,,crime of war" und ,,war crimes" hervorgehoben, wie sie die Nürnberger und Tokyoter Rechtsprechung herausgearbeitet und die Anerkennung der Staaten gefunden hat, gleichzeitig aber die Unmöglichkeit betont, ,,crime of war“ gegenüber anderen als den Verliererstaaten zu ahnden. Zum Problem der Legimität des amerikanischen Counterinsurgency in Vietnam, das die Nürnberger Prinzipien seit ihrer Verkündung der stärksten Belastungsprobe ausgesetzt hat, meint Warnke, daß die politische Sanktion die angemessene Reaktion auf Geschehnisse sei, die mehr auf politisch-historischen Verwicklungen basieren und nicht zugleich als Verstöße gegen anerkannte Kriegsführungsregeln identifiziert werden können. Warnke läßt jedoch keinen Zweifel daran, daß, unabhängig vom Nachweis der Gerechtigkeit eines Krieges oder vom Bestehen eines nationalen Konsenses in dieser Frage, die individuelle Verantwortlichkeit für begangene Kriegsverbrechen in jedem Fall fortbestehe. Dies gelte für Befehle ausführende Kombattanten ebenso wie für deren Kommandeure. In diesem Zusammenhang wird an die Verurteilung des Generals Yamashita in Tokyo erinnert, der sich mit der Verteidigung, er habe keine Kenntnis von den Verbrechen, die seine Truppen auf den Philippinen begangen hätten, und auch keine Kontrollmöglichkeiten gehabt, sich nicht vor dem Strang retten konnte.

Einen extremen Standpunkt in der Frage der individuellen Verantwortlichkeit des Kombattanten nimmt Richard A. Wasserstrom ein. Er argumentiert mit Telford Taylor auf der Basis der militärischen Notwendigkeit zugunsten einer nahezu totalen Freistellung des Einzelnen, in die der Gefangenenmord ebenso einbezogen wird wie das bewußte Bombardement 
ziviler Ziele - Frauen, Kinder, Greise eingeschlossen. Seine Argumentation ist so extrem, $\mathrm{da} ß$ sich eine Auseinandersetzung mit ihr nicht lohnt. Wasserstroms Thesen sind jedoch dort des Nachdenkens wert, wo er auf die Unbestimmtheit und Irrationalität zahlreicher Regeln des ius in bello und auf ihre uneinheitliche Praxis hinweist, die es dem einzelnen Kombattanten oft als unzumutbar erscheinen lassen, von klaren Befehlen, selbst wenn sie unter Verstoß gegen anerkannte Kriegsgesetze ergehen, abzuweichen. Insofern ist Wasserstrom der einzige Autor des Sammelbandes, der zu der Kardinalfrage der subjektiven Möglichkeiten des Kombattanten, die Kriegsregeln einzuhalten, zumindest im Ansatz Stellung nimmt.

Demgegenüber will Leonard B. Boudin eine strafrechtliche Immunität des Kombattanten für Kriegshandlungen, die auf illegale Befehle zurückgeführt werden können, nicht anerkennen, und zwar unter Hinweis auf Lauterpacht selbst dann nicht, wenn damit das eigene Leben gefährdet werde. Umgekehrt schaffe das Dilemma, vor dem militärische Führer stehen, die über die militärische Disziplin in gleicher Weise wie über die Einhaltung der Gesetze des Krieges zu wachen haben, keine Priorität der militärischen Belange. Vielmehr stehen beide Notwendigkeiten in einem untrennbaren Zusammenhang. Zu Recht erklärt deshalb Boudin unter Hinweis auf Taylor: Je stärker dem Soldaten gegenüber auf Beachtung des Gehorsams geachtet wird, desto größer ist die Haftung des Offiziers, daß seine Anordnungen auch im Einklang mit dem Recht stehen.

Zuletzt sei noch ein Wort zur Art und Weise gesagt, in der die Autoren dieses Sammelbandes die Diskussion geführt haben, weil dies nicht unwesentlich erscheint für die Frage, in welche Richtung und mit welcher Intensität sich die Kriegsführungsregeln im bewaffneten Konflikt fortentwickeln werden. Die Debatte kontroverser Positionen in einer Frage, die das amerikanische Selbstverständnis zutiefst betroffen hat, wird mit bemerkenswerter Offenheit und Sachlichkeit geführt, was die öffentliche Meinung in den USA als eine funktionierende Demokratie ausweist, der Debatte aber zugleich einen hohen Legitimitätsanspruch in der Sachaussage gibt. Dennoch bleibt der Eindruck bestehen, daß die konkreten Fragen, trotz ihres aktuellen Bezuges, viel zu intellektuell erörtert werden und nicht emotional genug auf ihre Konsequenzen hin überprüft werden. Der Grund dafür liegt, wie es scheint, in den mangelnden einschlägigen Erfahrungen der Autoren, sowohlihrer selbst als auch als Teil einer Nation, die auf eigenem Boden bewußt keinen Krieg erlebt hat. Wer selbst nicht durch den Feuersturm eines Flächenbombardements hindurchgegangen ist oder einen Napalmangriff nicht selbst als Betroffener erlebt hat, ist weniger in der Lage, die Frage der Zulässigkeit von Waffensystemen und des Schutzes von Nichtkombattanten adäquat zu gewichten und zu beantworten. Damit soll nicht gesagt sein, daß die eigene Erfahrung conditio sine qua non jeder derartigen Erörterung sei. Im Laufe der Geschichte haben aber über den humanitären Fortschritt bei der Austragung bewaffneter Konflikte nicht diejenigen entschieden, die das Problem intellektuell zu bewältigen suchten. Dies gilt für Francisco de Vitoria, der die ersten Voraussetzungen für ein Völkerrecht auf der Grundlage der Humanität schuf, und dies gilt für Henry Dunant, ohne dessen engagiertes Eintreten für den Einzelnen dieHaager Kriegführungsregeln nicht zustande gekommen wären. In bestimmten Grenzen darf dies auch Richard Falk für sich in Anspruch nehmen.

Hermann Weber 